\title{
Bilingual Embeddings with Random Walks over Multilingual Wordnets
}

\author{
Josu Goikoetxea ${ }^{\dagger}$, Aitor Soroa, Eneko Agirre \\ IXA NLP group, Faculty of Informatics, UPV/EHU, \\ Manuel Lardizabal 1 (20018), Donostia, Basque Country
}

\begin{abstract}
Bilingual word embeddings represent words of two languages in the same space, and allow to transfer knowledge from one language to the other without machine translation. The main approach is to train monolingual embeddings first and then map them using bilingual dictionaries. In this work, we present a novel method to learn bilingual embeddings based on multilingual knowledge bases (KB) such as WordNet. Our method extracts bilingual information from multilingual wordnets via random walks and learns a joint embedding space in one go. We further reinforce cross-lingual equivalence adding bilingual constraints in the loss function of the popular skipgram model. Our experiments involve twelve cross-lingual word similarity and relatedness datasets in six language pairs covering four languages, and show that: 1) random walks over multilingual wordnets improve results over just using dictionaries; 2) multilingual wordnets on their own improve over text-based systems in similarity datasets; 3) the good results are consistent for large wordnets (e.g. English, Spanish), smaller wordnets (e.g. Basque) or loosely aligned wordnets (e.g. Italian); 4) the combination of wordnets and text yields the best results, above mapping-based approaches. Our method can be applied to richer KBs like DBpedia or BabelNet, and can be easily extended to multilingual embeddings. All software and resources are open source.
\end{abstract}

Keywords: multilinguality, distributional semantics, embeddings, random walks, WordNet

\footnotetext{
${ }^{\dagger}$ Corresponding author. Email adress: josu.goikoetxea@ehu.eus
} 


\section{Introduction}

The use of distributional semantic models in the Natural Language Processing community has grown substantially over the last few years [1]. In particular, unsupervised distributional models such as skipgram [2] and GloVe [3] represent semantic and syntactic features of words in low dimensional embeddings learned using co-occurrence information from corpora. These word embeddings have been very effective when addressing a wide variety of tasks, such as word similarity [4], word analogy [5, 2], sentiment analysis [6], document classification [7] and natural language inference [8], to name a few.

Several authors [9, 10, 11, 12, 13] have proposed methods to combine textbased distributional information with the semantic information contained in Knowledge Bases (KB) such as WordNet[14]. The combination improves the results over corpus-only methods, showing that text and knowledge bases contain complementary information. Our work can be seen as an extension of those ideas to a bilingual setting.

Given the success of distributional models on monolingual tasks, researchers have also trained bilingual word embeddings that represent words in different languages within the same vector space. The approaches can be classified according to the bilingual resources used. Some methods require large parallel corpora [15, 16], which is a scarce resource for many language pairs. Other methods use more widely available document-aligned corpora [17, 18, 19]. Finally, other alternative approaches rely on bilingual dictionaries to learn a linear transformation that maps independently-learned monolingual embeddings [20, 21, 22]. The latter setting only requires monolingual corpora and a small bilingual dictionary (around 5,000 word pairs), which can be obtained easily for many language pairs.

We chose the latter setting for this article, but, instead of bilingual dictionar- 
ies, we turn our attention to multilingual wordnet:1 [23]. Bilingual dictionaries are basically pairs of translation-equivalent words, while bilingual wordnets also contain a wealth of information about the structure of the lexicon, including semantic relations like synonymy, hypernymy (aka is-a), meronymy (aka part-of), antonymy, and gloss-relations [14]. Our hypothesis is that the structural information in wordnets can be exploited to produce better bilingual embeddings, compared to methods that just use the word pairs in bilingual dictionaries.

The main contribution of this article is thus a novel method based on random walks over concepts in the multilingual KBs to produce bilingual embeddings: each time the random walker arrives at a concept, it emits, at random, one possible lexicalization in any of the two languages. These emitted words constitute a bilingual synthetic corpus which encodes, via bilingual co-occurrences, the latent structure of the bilingual wordnet. We feed the bilingual co-occurrences into the skipgram model [1], so that words in the two languages that are structurally similar in the KB have similar embeddings. A similar strategy was previously shown to be very effective for monolingual wordnets [24], and we now extend it to bilingual wordnets with excellent results. The proposed method is independent of the particular KB used, and other multilingual KBs such as Dbpedia or BabelNet [25] can be considered.

In addition, we also explore the use of bilingual constraints extracted from wordnets in the loss function of skipgram. These constraints act as regularizers, implementing the notion that two words which are translations of each other (according to the bilingual wordnet) should have embeddings which are close in embedding space. This can be seen as an extension of [9, 12] to the bilingual case.

In order to complement the WordNet structure with distributional information, we combine the bilingual synthetic corpus with monolingual text corpora,

\footnotetext{
${ }^{1}$ We follow the literature using capitalized WordNet for the English version and wordnets in lowercase to refer to instantiations in other languages. As we focus on two languages, we will talk about bilingual wordnets, although they are truly multilingual.
} 
and then learn bilingual embeddings using skipgram in one go. This method, coupled with the bilingual constraints, improves the state-of-the-art in twelve cross-lingual similarity and relatedness datasets comprising five language pairs and four languages.

The article is structured as follows. We start presenting related work. Section 3 presents the skipgram model and the modified loss function which includes bilingual constraints. Section 4 presents the random walk method for learning embeddings from bilingual knowledge bases. Section 5 presents the experiments on cross-lingual word similarity and relatedness, followed by a summary and comparison to the state-of-the-art. Finally, we draw the conclusions and future work.

\section{Related work}

Several methods to learn word embeddings from text corpora exist, being skipgram [2], continuous bag of words [2] and GloVe [3] the most popular. Without lose of generality, in this work we have chosen the most widely used, skipgram, although the techniques here presented can be easily applied to other embedding learning models.

Following the hypothesis that text and knowledge bases contain complementary semantic information, several authors have tried to enhance the quality of distributional representations by incorporating information from knowledge bases. 10] proposed a method to infuse relational information from WordNet [14], Paraphrase Database [26], FreeBase [27] and FrameNet 28] to embeddings learned from corpora, improving significantly the results in word similarity tasks.

Some other authors learn embeddings jointly using knowledge base relations and corpus co-occurrences. [29] implemented a LSA-based multiview model using several semantic resources. [30] introduced relational information from FreeBase in the learning process and performed projections of entity embeddings onto an hyperplane w.r.t. the knowledge base relation. [9] and [12] incorporated WordNet information introducing a regularizer in the loss function 
which constrained the neighbors in the knowledge base to have similar representations. In this article we have extended the latter technique, incorporating bilingual constraints extracted from bilingual wordnets into the skipgram loss function (see section 3.2).

In a different strand of work, 24] learn knowledge-based embeddings using graph-based methods over WordNet; they performed random walks over WordNet relations to create a synthetic corpus that implicitly encoded the structure of the knowledge base, similar to the DeepWalk method [31]. In later work, they show that the synthetic corpus can be combined with text corpora, yielding embeddings which combine both sources of information, and surpassing other methods in word similarity tasks [13]. In this work we extend the random walk algorithm to work with bilingual wordnets, both to produce knowledgebased bilingual embeddings, and hybrid text- and knowledge-based bilingual embeddings (see Section 4).

Bilingual distributional representations have also attracted a lot of attention, and researchers have addressed the training of bilingual embeddings using several approaches [20, 16, 15, 17, 22, 21, 19, 18, 32, 33, 34]. Many methods require bilingual corpora aligned at the document, paragraph or sentence level [15, 16, 17, 18, 19], which can be difficult to obtain, particularly when dealing with low resource languages. In this work we focus on methods that rely on monolingual corpora and relatively small bilingual dictionaries (containing ca. 5000 pairs) 201, 21, 22], which are more widely available. A common strategy of these methods is to exploit structural similarities among separate vector spaces. For instance [20, 32], started from two monolingual spaces, and then learned a linear transformation from the source embedding space to the target embedding space that minimizes the eucledian distance of the word pairs in the bilingual dictionary. This approach was improved by 33], who constrained the transformation matrix to be orthogonal. Further, [22] added length normalization and the maximization of the cosine similarity, forcing the orthogonality constraint to keep the normalization after the mapping. While the latter authors learned a single transformation from target to source language, 34] used canonical cor- 
relation analysis to learn separate transformations, projecting the monolingual spaces into a shared space. More recently, [21] presented a framework that generalized previous approaches and showed the common aspects among them. Their method includes orthogonality constraints, a global preprocessing with length normalization and dimension wise mean centering, achieving the best accuracy to date in the dictionary induction task. We will use this latter method as a baseline. Note that these methods learn monolingual embeddings first, and then map those embeddings. Our method learns a joint bilingual space in one go.

Finally, NASARI [35] combines text-based and knowledge-based methods, yielding multilingual vector representations. They exploit BabelNet [25], a large multilingual semantic network that includes, among others, information from wordnets, Wikipedia, OmegaWiki and Wiktionary. They learn representations for BabelNet concepts with a method that analyzes the content words in Wikipedia articles, weighting them using a schema similar to $t f-i d f$, and mapping each word to its candidate senses according to BabelNet. As a result, each BabelNet concept is represented by a vector indexed by language-independent concepts, with excellent results in cross lingual similarity experiments. Although NASARI uses a richer knowledge base (BabelNet), we will compare the performance of NASARI head-to-head our method, which only uses wordnets.

\section{Bilingual embeddings via constraints}

In this section we describe our method to learn bilingual embeddings using constraints. We start by reviewing the general skipgram model and then describe the extension to infuse information from bilingual dictionaries as regularizers in the loss function .

\subsection{Skipgram}

The skipgram model [1] uses the current word $w$ to predict the surrounding window of context words $c$. The parameters $\theta$ that maximize $p(c \mid w ; \theta)$ are 
learned from a text corpus. We will focus on the negative sampling implementation, where the loss function rewards the estimate of the probability for words that co-occur with each other, while penalizing the estimate of the probability for random word pairs co-occurring with each other. Negative sampling has shown to be a very effective alternative to the computationally expensive softmax, achieving state of the art results in many NLP tasks and substantially speeding up the learning process.

Skipgram assigns two vector 3 to each word in the vocabulary, which represent its semantic features as a target word and as a context word. In this article we will refer to the word and context vector spaces as $W$ and $C$ respectively, which are the parameters $\theta$ to be learned. Both $W$ and $C$ are $|V| \times D$ size matrices, being $|V|$ the size of the vocabulary and $D$ the dimensionality of the vectors. Equation 11describes the loss function for each word-context occurrence in the corpus according to the skipgram model with negative sampling, being $w \in W$ the target word, $c \in C$ a context word and $c_{n} \in C$ a random negative sample (indexed by $n$ ) drawn from a noise distribution $P(c)$.

$$
J_{s g}(w, c)=\log \left(\sigma\left(c^{t} w\right)\right)+\sum_{n=1}^{N} \mathbb{E}_{c_{n} \sim P(c)}\left[\log \left(\sigma\left(-c_{n}^{t} w\right)\right)\right]
$$

The total loss is the summation of Eq. (1) for all pairs of words $w$ and $c$ co-occurring in the corpus, as extracted using a context window of size $K$. The model has, among others, hyperparameters $K$ (context window size) and $N$ (number of negative samples). Stochastic gradient descent is used to find the parameters $W$ and $C$ that maximize the loss function in the corpus.

The embeddings learned by skipgram have proven to be very effective modeling semantic and syntactic phenomena. For instance, the word vectors in space $W$ have been used to model word equivalence and similarity, using the cosine of the vectors of two words to capture how similar the words are [4]. Evaluation

\footnotetext{
${ }^{2}$ The actual formula has been shown to be slightly different, but this is not relevant for the present work. We refer the interested reader to [5] for more details

${ }^{3}$ In the context of this article, we use word vectors and word embeddings interchangeably.
} 
of the word embeddings in $W$ in standard tests against human-curated gold standards has confirmed their quality [4]. In addition to using the embeddings in $W$ alone, other work has shown that both $W$ and $C$ contain complementary information [3, 36]. Following their work, we represent a word as the addition of the respective two vectors in $W$ and $C$, as preliminary experiments confirmed that these representations were more effective than using $W$ or $C$ alone.

\subsection{Introducing bilingual constraints}

Given (unaligned) monolingual corpora in two languages, a straightforward method to obtain bilingual embeddings is to merge and shuffle the corpora at a sentence level, and then to use skipgram to learn embeddings on the merged corpus. The efficiency of this method critically depends on how strings are shared across languages (e.g numbers, proper names or loan words), as these shared strings act as bridges that allow gathering bilingual co-occurrences [37]. Unfortunately, the number of such shared strings is in general too small, and translation equivalents tend to be distant in the embedding space, as we show empirically in the experimental section.

One way to reinforce translations in the joint embedding space is to include a regularization term in the loss function (Eq. 1) that forces embeddings of translation equivalences to eventually become closer in the joint vector space. This regularization term was first introduced in [9] and [12] to infuse information derived from external sources into the learning process (synonyms, related words, etc.), and we apply it here to deal with translations gathered from a bilingual dictionary.

Equation 2 shows the new loss function for each word occurrence $w$, which contains two terms: the loss function for co-occurrence pairs in Equation (1), and the regularizer which minimizes the $\mathrm{L} 2$ norm between $w$ and its translation equivalents $w_{l m}$ according to $\lambda \in \mathbb{R}^{+}$, the regularization coefficient. Translation equivalents for a word are indexed by $l$ (language) and $m$ (which is one of multiple $M_{l}(w)$ equivalents in that language). We also use monolingual constraints, where $l$ is the same language as that of word $w$. In this case we obtain syn- 
onyms of $w$ instead of translations equivalents. Both monolingual and bilingual constraints are extracted from wordnets (cf. section 5.1).

$$
J_{s g+}(w)=\sum_{c \in \text { window }_{K}(W)} J_{s g}(w, c)-\lambda \sum_{l=1}^{2} \sum_{m=1}^{M_{l}(w)}\left\|w-w_{l m}\right\|_{2}^{2}
$$

\section{Random walks over knowledge bases}

As mentioned in the related work section, one of the most effective methods to build word representations from knowledge bases is to perform random walks over the knowledge base graph to collect co-occurrence data which reflects the structure of the knowledge base [24]. Given the co-occurrence information, skipgram is used to produce embeddings that encode the meaning of words according to the knowledge base. Those co-occurrences, and derived embeddings, can be seen as the knowledge-based counterpart of text co-occurrences, and they characterize the meaning of a word from the perspective of the relations in the knowledge base. We will first present this method, and then extend it to represent words in two languages in the same embedding space.

\subsection{Monolingual random walks}

Let's model the target knowledge base as a graph $G=(C, E)$, being $C$ the list of vertices (concepts) and $E$ the edges between concepts (relations in the knowledge base). In addition, let $N(c)$ be the set of neighbors of node $c$ in the graph, and let $D(c)$ be the possible lexicalizations of concept $c$.

Random walks are simulated by means of a Monte Carlo method, as follows. The algorithm starts at one random concept from $C$. Every step of the walk the algorithm tosses a coin: with probability $\alpha$ a neighbor concept is selected following one edge at random; with probability $1-\alpha$ the walk is halted and a new concept from $C$ is chosen at random. Every time a concept is selected, a possible lexicalization is chosen and printed. Every time the walk is halted, a new context is produced (that is, a newline is printed). The algorithm stops once it reaches a pre-fixed number of walks $I$. The algorithm has thus two hyperparameters, 


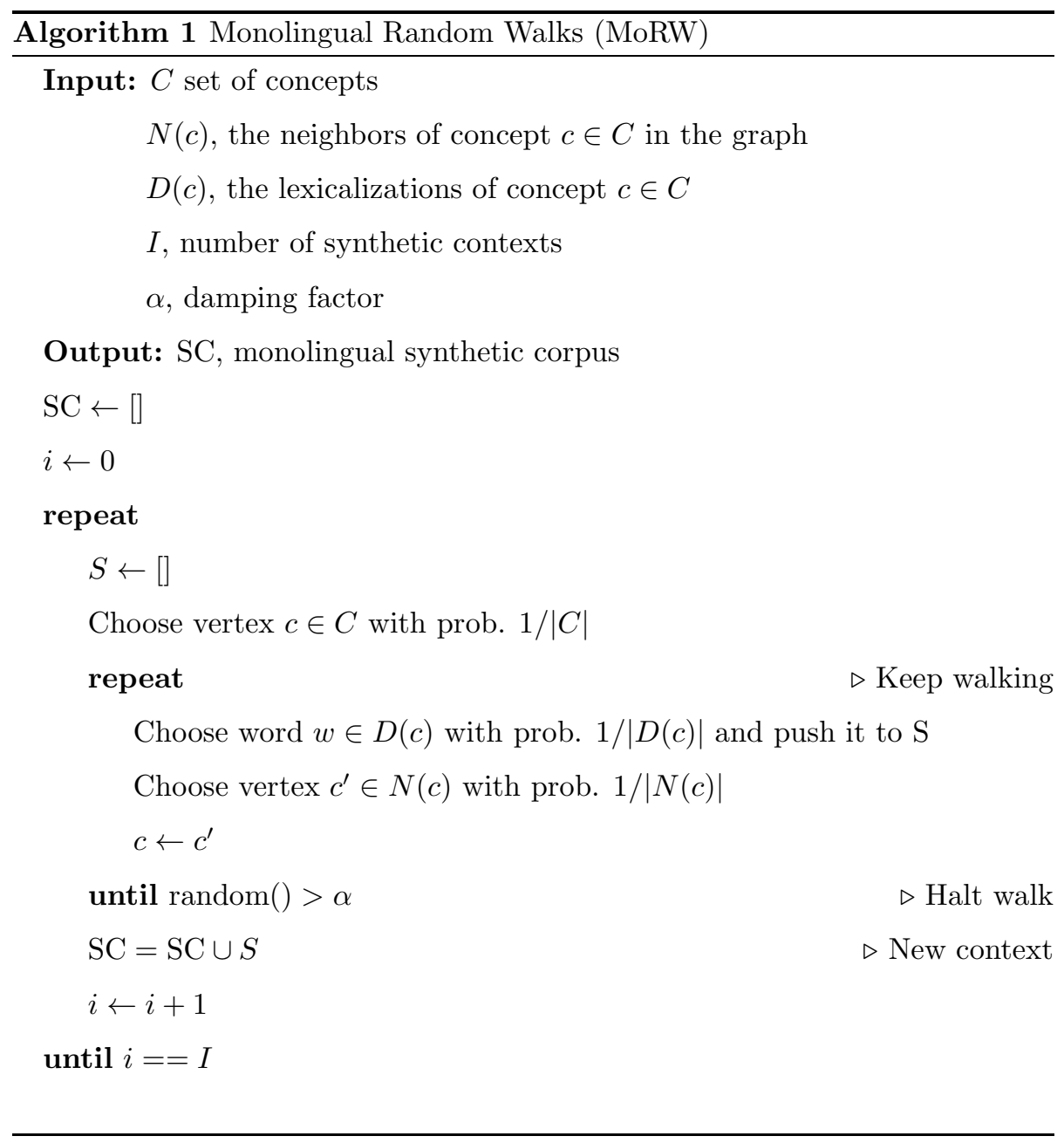

$\alpha$ and $I$. All probability distributions and random choices, except $\alpha$, follow the uniform distribution. Algorithm 1 summarizes the procedure to perform monolingual random walks.

For instance, a synthetic context created out of WordNet looks like the following:

$$
\text { paw feline felid ounce panthera lion sekhet }
$$

The latter context has been produced using WordNet 3.0 with gloss relations and $\alpha=0.85$. The lexicalization for the first vertex is paw, a soft foot 
of mammals which normally has claws; the walk continues following an edge to the more abstract feline, a subfamily of cats that includes mostly small to medium-sized cats; follows another edge to the also abstract felid, the family of cats that the previous word feline belongs to; follows a hyponymy edge to ounce, a long haired leopardlike feline of the mountains of central Asia, also known as snow leopard; then comes panthera, the genus that ounce belongs to; follows another edge to the concept lexicalized by lion, also related to the genus panthera; continues with the Egyptian lion headed goddess sekhet via a gloss relation, and finally halts the walk. The algorithm would continue until the desired number of contexts $I$ is obtained.

This synthetic corpus alone is a good resource to learn embeddings from, as it leverages the structural information of WordNet in the form of word cooccurrences in the synthetics corpus, and provides excellent results in monoingual word similarity tasks [24]. But, given the complementarity of the information in knowledge bases and text corpora, it is possible to combine knowledgebased and distributional information with excellent results [13]. We follow their method, which concatenates and shuffles the synthetic corpus with natural text corpora at the sentence level, and then learns embeddings from this hybrid corpus. For instance, the method would mix co-occurrences from the above example for panthera with those of naturally occurring text as the following:

Felids of the genus Panthera (tigers, lions, jaguars and leopards) also produce sounds similar to purring.

\subsection{Bilingual random walks}

The method for generating a bilingual synthetic corpora is the same as for the monolingual case, but, when arriving to a concept, a lexicalization in any of the two languages is emitted at random. The method is formalized in Algorithm 2 , where $C$ and $N(c)$ are defined as before, and $D_{l}(w)$ is the set of all lexicalizations of concept $c$ in language $l \in\left\{l_{A}, l_{B}\right\}$. Note that $D_{l}(c)$ can be empty when the concept is not lexicalized in that particular language, due to different coverages 
of the wordnets or lexicalization differences across languages. Algorithm 2 thus controls for this case and also for potentially empty contexts.

There are currently open wordnets in thirty-four languages 4 . Some of them have been created following the original English structure (expand approach), where the concepts are lexicalized in more than one language and the relations between concepts are kept untouched. Other wordnets have been created separately (merge approach), with different sets of concepts and relations, and a mapping to the English WordNet is provided. In the experiments (cf. Section 5) we will use the English WordNet, two expand-approach wordnets (Basque and Spanish) and one merge-approach wordnet (Italian). Note that the wordnets vary in size, with English having the largest and Basque the smallest (see Table 2).

Wordnets created using the expand approach share the concept inventory and the relations between those concepts, and thus cross-lingual information is fully compatible. Ideally, all concepts should be lexicalized in both languages, but in reality only a fraction of the concepts is lexicalized in both languages, depending on the sizes the wordnets involved. In the merge approach, a mapping between wordnets is necessary to find equivalent concepts and relations across languages. In the case of the Italian WordNet, we added all concepts of the Italian and English wordnets to the graph, as well as the monolingual relations in each wordnet, plus the mapping between Italian and English concepts.

The next example shows a bilingual synthetic context created for English and Basque (in bold) which includes panthera:

elur-pantera panthera felidae sabertooth smiledon ugaztun erignathus

The example starts with the Basque word elur-pantera (snow leopard); then jumps to the more general lexicalization in English panthera, remains in the same language with the even more general felidae and goes more specific again in sabertooth and smiledon; in the next step it emits the abstract Basque word

\footnotetext{
${ }^{4}$ http://compling.hss.ntu.edu.sg/omw/
} 


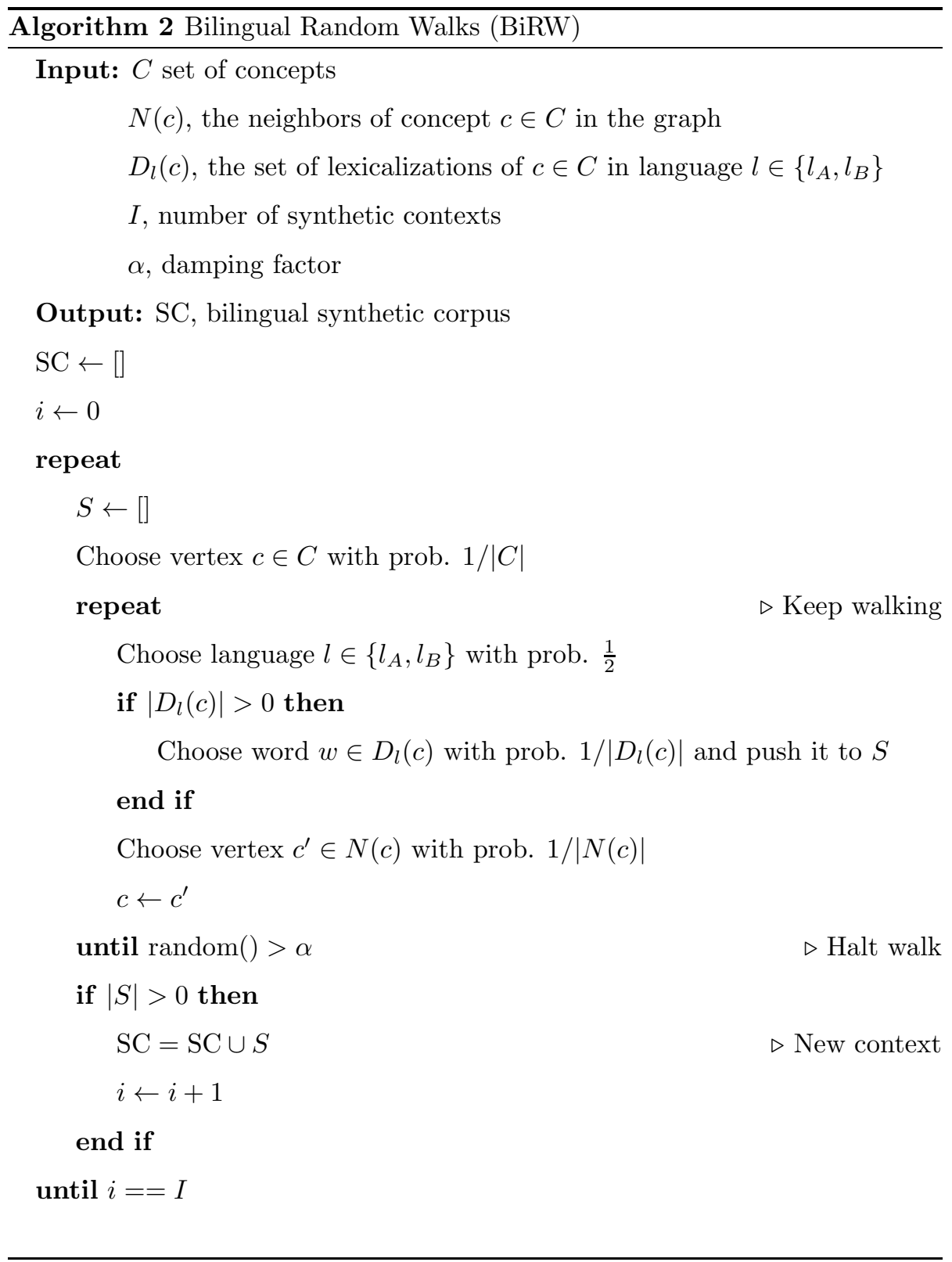


ugaztun (mammal in English); the walk halts in erignathus, a term that refers to the family of seals. The unbalance of lexicalizations in Basque and English is reflected in the bilingual random walks, with more lexicalizations available for English.

Similar to monolingual random walks, the bilingual random walks can be combined with monolingual text corpora, producing a bilingual hybrid corpora which mixes bilingual co-occurrences from bilingual random walks and monolingual co-occurrences from the monolingual corpora. We will see that this is an effective method to produce bilingual embeddings.

\section{Experiments}

Word similarity and relatedness are the most common evaluation methods to measure the quality of monolingual embeddings [38, 39, 4, 36, 10], and we thus chose cross-lingual word similarity and relatedness to measure the quality of bilingual word embeddings. In cross-lingual word similarity and relatedness, given a set of pair of words in two languages, the goal is to return scores which are close to human-curated gold standard ground truth scores. Given two words, the automatic score is obtained by computing the cosine similarity of the corresponding embedding vectors. The evaluation measure is typically Spearman's rank correlation 5 .

We reviewed existing word similarity and relatedness datasets in a number of languages, with English having the highest amount of datasets. In order to select the target languages we took into account the following factors: the existence of at least two datasets for the language (see next Section) and the existence of a sizeable open wordnet6. The overlap yielded three languages, English, Italian and Spanish, two romance and one west germanic language. In order to add more linguistic diversity, we selected Basque, an agglutinative language which is a language isolate (no related language is known). There is a sizeable wordnet

\footnotetext{
${ }^{5}$ https://en.wikipedia.org/wiki/Spearman\%27s_rank_correlation_coefficient

6 http://compling.hss.ntu.edu.sg/omw/
} 


\begin{tabular}{|l|r|}
\cline { 2 - 2 } \multicolumn{1}{c|}{} & \multicolumn{1}{|c|}{ Tokens } \\
\hline EU & $160 \cdot 10^{6}$ \\
IT & $380 \cdot 10^{6}$ \\
ES & $430 \cdot 10^{6}$ \\
EN & $1,700 \cdot 10^{6}$ \\
\hline
\end{tabular}

Table 1: Amount of tokens per monolingual corpora, in ascending order.

for Basque, and we manually annotated two datasets. As we will see in detail below, our experiments involve four languages and twelve cross-lingual datasets in six language pairs: English-Basque (ENEU), English-Spanish (ENES), EnglishItalian (ENIT), Spanish-Basque (ESEU), Spanish-Italian (ESIT) and ItalianBasque (ITEU).

\subsection{Experimental setting}

In order to learn the embeddings for the target languages we have used readily available Wikipedia corpora 7 , except for Basque, which was not available. The Basque Wikipedia corpus was extracted from the 07/04/2016 dump, and, given its smaller size, it was complemented with the Elhuyar Web Corpus [40], kindly made available from the authors on request. As Basque is an agglutinative language, we have stemmed the Basque corpus, but the rest of the corpora were not. All corpora were converted to lowercase. The sizes of the monolingual corpora are shown in Table 1 .

Regarding wordnets, we have used the Basque, English and Spanish open wordnets corresponding to the Multilingual Central Repository 3.0 [41]. Both the Basque and Spanish wordnets reuse the synset: 8 , hierarchy and relations from the English WordNet 3.0 version, including the gloss relations 9. For Italian we used ItalWordNet, which has its own set of synsets and relations [42], and

\footnotetext{
'http://linguatools.org/tools/corpora/wikipedia-monolingual-corpora/

${ }^{8}$ In WordNet, concepts are referred to as synsets. We use both terms interchangeably in this article.

${ }^{9}$ http://wordnet.princeton.edu/glosstag.shtml
} 


\begin{tabular}{|c|r|r|}
\cline { 2 - 3 } \multicolumn{1}{c|}{} & \multicolumn{1}{c|}{ Words } & Synsets \\
\hline EU & 26,701 & 30,464 \\
IT & 46,679 & 49,515 \\
ES & 53,039 & 55,814 \\
EN & 147,306 & 136,334 \\
\hline
\end{tabular}

Table 2: Number of words and synsets in each wordnet, in ascending order.

\begin{tabular}{|c|c|c|c|l|c|c|}
\hline \multicolumn{4}{|c|}{ monolingual } & \multicolumn{2}{c|}{ bilingual } & total \\
\hline EU & 24,985 & IT & 33,608 & EUIT & 109,031 & 167,624 \\
EU & 24,985 & ES & 43,218 & EUES & 67,685 & 135,888 \\
ES & 43,218 & IT & 33,608 & ESIT & 126,149 & 202,975 \\
EN & 152,219 & EU & 24,985 & ENEU & 99,861 & 277,065 \\
EN & 152,219 & IT & 33,608 & ENIT & 173,126 & 358,953 \\
EN & 152,219 & ES & 43,218 & ENES & 176,873 & 372,310 \\
\hline
\end{tabular}

Table 3: Amount of pairwise constraints per language pair, in ascending order. For each language pair, we report number of monolingual and bilingual constraints alongside the total.

includes links to the English WordNet 3.0. All are available from the Open Multilingual WordNet websit 10 . Table 2 reports the number of words and synsets in each wordnet. All lexicalizations have been lowercased for compatibility with the corpora.

In order to produce the bilingual constraints, we mined the dictionaries in the wordnets. That is, for each entry in the vocabulary, we checked its synsets and produced all possible lexicalizations in the same language (monolingual synonyms) and bilingual translation equivalents. Table 3 reports the number of pairwise constraints for each language pair.

Regarding the evaluation datasets, the available cross-lingual datasets were the cross-lingual counterparts of the following well-known monolingual

\footnotetext{
${ }^{10}$ http://compling.hss.ntu.edu.sg/omw/
} 
datasets: a word relatedness dataset, WordSim353 (WS) [43], and two similarity datasets, SimLex999 (SL) [44] and RG [45]. In order to build the crosslingual datasets, we followed the procedure proposed by [46], which combines two monolingual versions of the same dataset to produce a cross-lingual dataset. The monolingual versions that we used to construct the cross-lingual datasets are the following: the Italian versions of WordSim353 and SimLex999, as well as the RG version in Spanish created by [46]; the Spanish WordSim353 and SimLex999 presented by [47] and [48], respectively; the Basque WordSim353 and RG datasets produced in-house. We thus produced two datasets for each of the six language pairs, with the exception of English-Spanish, where we could produce three datasets, and Italian-Basque, with one common dataset, totaling twelve datasets.

We did not fit skipgram hyperparameters, and reused the parameters reported by [13] for calculating embeddings and the same regularization coefficient $\lambda$ as in [9]. In summary, dimensionality of 300, window size of 5 , number of negative samples of 5 , sub-sampling threshold of zero, and regularization coefficient $\lambda$ of 0.01 . The damping factors for random walks was set to $\alpha=0.85$ following [24].

\subsection{Results using bilingual constraints on text corpora}

In the first batch of experiments we focus on genuine text corpora, and check whether our method to learn embeddings jointly from monolingual corpora with bilingual constraints from wordnets is effective (JOINTC $\mathrm{txt}_{\mathrm{txt}}$ for short, cf. Section 3). We shuffled the sentences in the concatenation of two monolingual text corpora, yielding one bilingual corpus for each of the six language pairs, and then learned skipgram embeddings with the bilingual constraints extracted from the wordnets. Due to the unbalanced sizes of the corpora accross languages (cf. Table 1), we experimented with two different combinations: equal number of tokens in both languages (reducing the size of the largest corpus in the pair with random selection), or all tokens in both languages. Preliminary results showed that the first option, with balanced corpus sizes, produced comparable 
or better result:11, so all experiments of the joint method in this article have been performed with balanced corpora. Table 4 shows the sizes of the balanced merged text corpora. Note that the bilingual text in the merged corpora is not aligned at all, and that each sentence comes from one language. As a baseline, we also learned bilingual embeddings with no use of constraints, JOINT $\mathrm{txt}_{\mathrm{t}}$ for short.

We compare the results of our bilingual embeddings with a state-of-the-art method [21] ( MAP $\left._{\text {txt }}\right)$ which learns separate monolingual embeddings for each language using the corpora in Table $\left[\mathbb{1}^{2}\right.$, and then learns a mapping based on the bilingual dictionary extracted from the wordnets (cf. Section 2). We use the same bilingual dictionaries as in our method (cf. Table 3). The mapping is directional, we learned a mapping from the language with the smaller wordnet to the language with the larger wordnet, obtaining six mappings, one for each language pair. In order to compute the similarity between two words, as customary 21], we first apply the learned mapping to one of the words. For instance, for a pair with an English word and a Basque word, we compute the cosine between the mapped embedding of the Basque word and the embedding of the English word.

Table 5 reports the results for the six language pairs. The results of the baseline (JOINT txt) are good, even if the text in one language is independent from each other. This results agree with those reported by [37], who attributed the surprisingly good results to lexical borrowing, including numbers, loan words and named-entities, such as Hamlet or Mandela, that are shared across the two languages. It thus seems that the mere concatenation of texts already provides enough co-occurrence information to yield acceptable results when learning a joint embedding space.

\footnotetext{
${ }^{11}$ In addition, we also tried with larger English corpora totaling $5 \cdot 10^{9}$ tokens, with no improvement.

${ }^{12}$ Note that this method has access to more tokens than our method, as better results were observed with larger corpora.
} 


\begin{tabular}{|l|c|}
\cline { 2 - 2 } \multicolumn{1}{c|}{} & Tokens \\
\hline ENEU & $320 \cdot 10^{6}$ \\
ESEU & $320 \cdot 10^{6}$ \\
EUIT & $320 \cdot 10^{6}$ \\
ENIT & $760 \cdot 10^{6}$ \\
ESIT & $760 \cdot 10^{6}$ \\
ENES & $860 \cdot 10^{6}$ \\
\hline
\end{tabular}

Table 4: Amount of tokens per merged text corpus, in ascending order. Each bilingual corpus comprises $50 \%$ of monolingual sentences from each language.

\begin{tabular}{|l|l|rrr|rrr|r|r|}
\cline { 3 - 8 } \multicolumn{2}{l|}{} & WS & SL & RG & WS & SL & RG & \multicolumn{2}{|l|}{} \\
\hline \multirow{3}{*}{ ENEU } & MAP $_{\text {txt }}$ & $\mathbf{6 8 . 5}$ & - & $\mathbf{8 1 . 5}$ & 59.8 & $\mathbf{3 5 . 8}$ & - & MAP $_{\text {txt }}$ & \\
& JOINT $_{\text {txt }}$ & 52.8 & - & 58.3 & 57.6 & 24.9 & - & JOINT $_{\text {txt }}$ & ENIT \\
& JOINTC $_{\text {txt }}$ & 61.6 & - & 70.9 & $\mathbf{6 2 . 5}$ & 26.9 & - & JOINTC $_{\text {txt }}$ & \\
\hline \multirow{5}{*}{ ENES } & MAP $_{\text {txt }}$ & $\mathbf{6 2 . 6}$ & $\mathbf{3 3 . 9}$ & $\mathbf{7 9 . 5}$ & $\mathbf{5 5 . 9}$ & $\mathbf{3 1 . 6}$ & - & MAP $_{\text {txt }}$ & \\
& JOINT $_{\text {txt }}$ & 59.0 & 20.5 & 63.8 & 50.6 & 25.1 & - & JOINT $_{\text {txt }}$ & ESIT \\
& JOINTC $_{\text {txt }}$ & 62.5 & 26.9 & 71.2 & 52.4 & 28.7 & - & JOINTC $_{\text {txt }}$ & \\
\hline \multirow{3}{*}{ ESEU } & MAP $_{\text {txt }}$ & $\mathbf{6 4 . 4}$ & - & $\mathbf{6 5 . 1}$ & $\mathbf{6 0 . 9}$ & - & - & MAP $_{\text {txt }}$ & \\
& JOINT $_{\text {txt }}$ & 51.5 & - & 41.7 & 46.3 & - & - & JOINT $_{\text {txt }}$ & EUIT \\
& JOINTC $_{\text {txt }}$ & 57.5 & - & 49.0 & 51.5 & - & - & JOINTC $_{\text {txt }}$ & \\
\hline
\end{tabular}

Table 5: Results (Spearman) on the cross-lingual datasets using text corpora alone. MAPtxt refers to monolingual text vector spaces with mapping. JOINTtxt and JOINTCtxt refer to joint bilingual vector space, without and with bilingual constraints, respectively. Best result for each dataset and language pair in bold.

The method to infuse bilingual constraints (JOINTC $\mathrm{txt}_{\mathrm{t}}$ ) improves the results across all language pairs, showing that our method to modify the loss function of skipgram (cf. Section 3.2) is effective. In any case, the mapping method from 21] $\left(\mathrm{MAP}_{\mathrm{txt}}\right)$ yields the best results across the board, except for ENIT, where JOINTC $_{\text {txt }}$ obtains slightly higher results. This shows that, when using text corpora, the current state of the art method (learn embeddings independently and then map them) is more effective than our method to concatenate the corpus 
and use the dictionary as a constraint in the loss function to learn bilingual embeddings jointly. We will show, in the following sections, that the situation is reversed when dealing with knowledge bases.

Note that the results in Table 5 on the cross-lingual datasets are lower but close to those reported on monolingual datasets. For instance, [49] report results of 0.73 on WS and 0.83 on RG using skipgram, while the best results in Table 5 are 0.69 and 0.81 , respectively. Note that the numbers are not comparable, as the translations might increase the difficulty of the task 13 . When comparing accross language pairs, the results for some language pairs are lower, with ENEU reporting the best scores and ENIT and ENES similar values. The variability across languages can be explained by a number of factors, including different difficulty of the datasets because of polysemy in the translations. A study of those factors is out of the scope of this article. Our work focuses on comparing the performance of different systems, which is fine as long as their relative performance is coherent across language pairs and datasets.

\subsection{Results using random walks on KBs (synthetic corpora)}

In this set of experiments, we focus on the synthetic corpora produced using random walks over the wordnets, as described in Section 4 without access to text corpora. We learn bilingual embeddings using the three methods tested in the previous section, but applied to the synthetic corpora, as follows: the state of the art $\mathrm{MAP}_{\mathrm{kb}}$, and our two methods JOINT $\mathrm{kb}$ and JOINTC $\mathrm{kb}_{\mathrm{kb}}$. In the case of $\mathrm{MAP}_{\mathrm{kb}}$, we learned monolingual embeddings from monolingual synthetic corpora. In order to have a comparable numbers of tokens (with respect to $\left.\mathrm{MAP}_{\text {txt }}\right)$, we produced synthetic corpora of the same sizes as in Table 1 . The mapping is then performed using the dictionary extracted from the bilingual wordnet, as in the previous section. In the case of JOINT $\mathrm{kb}$ and $\mathrm{JOINTC}_{\mathrm{kb}}$, we produced bilingual synthetic corpora totaling the same number of tokens as in

\footnotetext{
${ }^{13}$ Note also that these figures were obtained using different corpora and hyperparameters, so they are only mentioned as a point of reference.
} 


\begin{tabular}{|c|c|c|c|c|c|c|c|c|c|}
\hline & & & & & & & \\
\hline & & WS & SL & $\mathrm{RG}$ & WS & SL & RG & & \\
\hline \multirow{3}{*}{ ENEU } & $\mathrm{MAP}_{\mathrm{kb}}$ & 65.1 & - & 88.2 & 55.8 & 47.2 & - & $\mathrm{MAP}_{\mathrm{kb}}$ & \multirow{3}{*}{ ENIT } \\
\hline & $\mathrm{JOINT}_{\mathrm{kb}}$ & 68.9 & - & 84.8 & 59.6 & 46.6 & - & $\mathrm{JOINT}_{\mathrm{kb}}$ & \\
\hline & JOINTC $_{\mathrm{kb}}$ & 67.6 & - & 86.1 & 59.8 & 46.7 & - & JOINTC $_{\mathrm{kb}}$ & \\
\hline \multirow{3}{*}{ ENES } & $\mathrm{MAP}_{\mathrm{kb}}$ & 62.9 & 46.1 & 77.1 & 44.9 & 38.5 & - & $\mathrm{MAP}_{\mathrm{kb}}$ & \multirow{3}{*}{ ESIT } \\
\hline & JOINT $_{\mathrm{kb}}$ & 64.5 & 46.6 & 79.2 & 50.4 & 42.7 & - & JOINT $_{\mathrm{kb}}$ & \\
\hline & JOINTC $_{\mathrm{kb}}$ & 64.7 & 48.2 & 79.7 & 49.1 & 42.7 & - & JOINTC $_{\mathrm{kb}}$ & \\
\hline \multirow{3}{*}{ ESEU } & $\mathrm{MAP}_{\mathrm{kb}}$ & 58.0 & - & 67.6 & 55.5 & - & - & $\mathrm{MAP}_{\mathrm{kb}}$ & \multirow{3}{*}{ EUIT } \\
\hline & $\mathrm{JOINT}_{\mathrm{kb}}$ & 60.1 & - & 66.6 & 49.2 & - & - & $\mathrm{JOINT}_{\mathrm{kb}}$ & \\
\hline & JOINTC $_{\mathrm{kb}}$ & 61.0 & - & 67.5 & 50.8 & - & - & JOINTC $_{k b}$ & \\
\hline
\end{tabular}

Table 6: Results (Spearman) on the cross-lingual datasets using synthetic, random-walk generated, corpora. $\mathrm{MAP}_{\mathrm{kb}}$ makes reference to results using separate monolingual random walk vector spaces plus the mapping. JOINT $\mathrm{kb}$ and $\mathrm{JOINTC}_{\mathrm{kb}}$ refer to the joint bilingual vector space, without and with bilingual constraints, respectively. Best result for each dataset and language pair in bold.

Table 4. As mentioned in Section 5, the different sizes of the wordnets causes the bilingual random walk corpora to contain more words in one language than in the other. For instance, in ENES around $65 \%$ of the tokens are in English, which raises to around $80 \%$ in ENEU and ENIT. Regarding the other languages, in ESEU and ESIT around $65 \%$ and $70 \%$ of the tokens are in Spanish, respectively, and in EUIT Basque amounts to $60 \%$ of the tokens. JOINTC $\mathrm{kb}_{\mathrm{kb}}$ uses the same bilingual constraints as $\mathrm{MAP}_{\mathrm{kb}}$.

Table 8 shows the performance on the six pairs of languages. The results for the three methods are mixed. In average, it seems all three methods do equally well, with each method winning in a comparable number of datasets. This means that, in the case of synthetic corpora, learning a joint space from the bilingual synthetic corpora matches the results of the method that first learns from monolingual synthetic corpora and then learns the mapping. The lack of improvement for constraints might be caused by the fact that the synthetic corpora implicitly encodes translations equivalents, and thus the added bilingual constraints are redundant, in contrast to the previous section. 
A comparison to the results of the best text-only embeddings ( $\mathrm{MAP}_{\mathrm{txt}}$ in Table 5 previous section) shows that the knowledge-based embeddings (all three methods in this section) perform consistently better on the similarity datasets by a large maring (RG and SL), while they underperform in the relatedness dataset (WS).

\subsection{Results using a combination of text and random walks (hybrid corpora)}

In these set of experiments, we combine textual corpora and synthetic corpora (we will refer to these as hybrid corpora, cf. end of Section 4.2), using the same methods as in the previous section. JOINThyb and JOINTC $\mathrm{Chb}_{\mathrm{hb}}$ are applied to the concatenation of the monolingual textual corpora and synthetic corpora produced using bilingual random walks over the wordnets. $\mathrm{MAP}_{\text {hyb }}$, on the other hand, learns the monolingual embeddings from two monolingual corpora which combine, each of them, monolingual text corpus and monolingual synthetic corpus. As in the previous sections, we use a dictionary extracted from the bilingual wordnet both for constraints and for learning the mappings.

In the case of JOINThyb and JOINTChyb we created hybrid bilingual corpora of the same size as the bilingual corpora in the previous sections (cf. Table 4) with a balanced number of tokens in the two languages, in order to control for corpus size and to show that the performance differences are not due to using larger corpora. As mentioned in Section 5, the different sizes of the wordnets causes the random walk corpora to contain more words in one language than in the other. In preliminary experiments we saw that balancing the number of tokens coming from genuine textual corpora and random walks produced better results. We thus have produced hybrid corpora which fulfill the following constrains: they contain the same number of tokens as those in Table 4, with a balanced number of tokens in each language (that is $50 \%$ each) and a balanced number of tokens between genuine and random walk corpora 14. The constraints

\footnotetext{
${ }^{14} \mathrm{We}$ are aware that other distributions across languages and genuine/random walk corpora are possible, but let the exploration of other options for future work.
} 


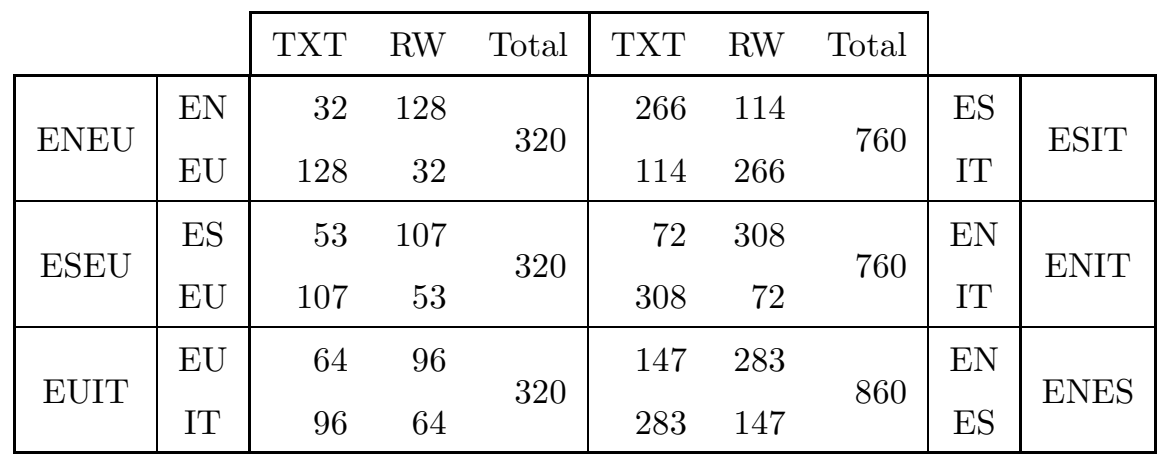

Table 7: Amount of tokens (in millions) in the six bilingual hybrid corpora, split according to language and source of the tokens (genuine textual - TXT - and random walks - RW).

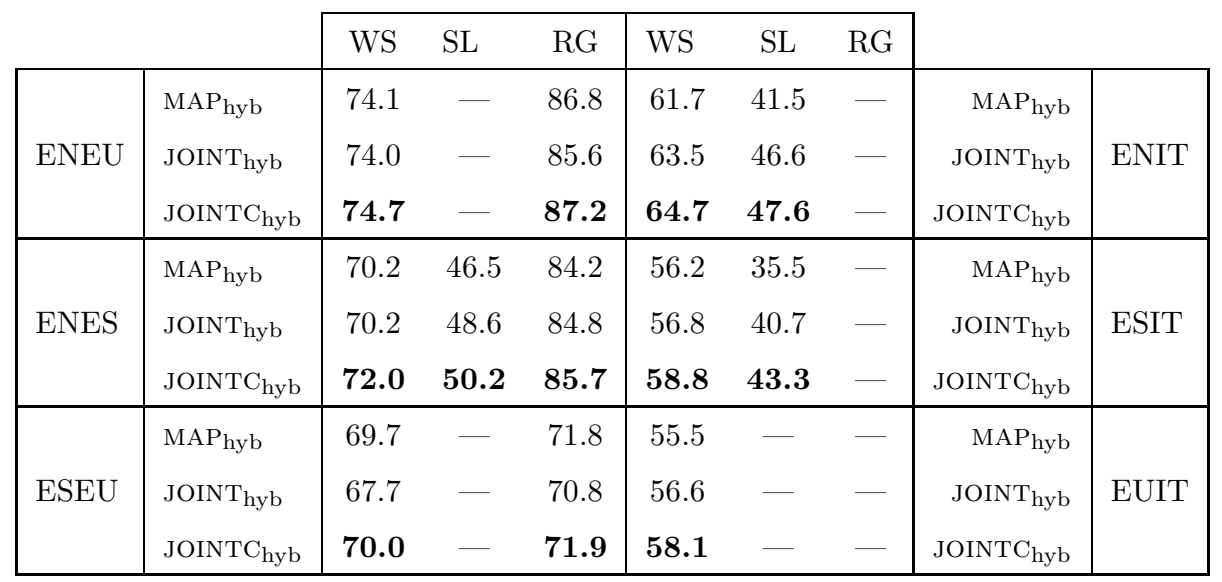

Table 8: Results (Spearman) on the cross-lingual datasets using hybrid corpora, combining natural and synthetic text. $\mathrm{MAP}_{\text {hyb }}$ makes reference to results using separate hybrid monolingual vector spaces plus the mapping. JOINThyb and JOINTC $\mathrm{Chb}_{\mathrm{h}}$ refer to the joint bilingual vector space, without and with bilingual constraints, respectively. Best results for each dataset and language in bold.

lead to the distribution of tokens shown in Table 7 .

Regarding MAPhyb, monolingual embeddings were learnt from two monolingual hybrid corpora which combined textual and synthetic corpora, with sizes following the following restrictions: balanced number of tokens for both languages, and balanced number of tokens for each source of corpus (genuine and random walks). The total numbers of tokens correspond to those in Table 4 . 


\begin{tabular}{|l|cccc|}
\cline { 2 - 5 } \multicolumn{1}{c|}{} & all & rel & sim & win \\
\hline MAP $_{\text {txt }}$ & $\underline{58.3}$ & $\underline{62.0}$ & $\underline{54.6}$ & $\underline{1}$ \\
JOINT $_{\text {txt }}$ & 46.0 & 53.0 & 39.1 & 0 \\
JOINTC $_{\text {txt }}$ & 52.2 & 57.8 & 46.6 & 0 \\
\hline MAP $_{\mathrm{kb}}$ & 58.2 & 55.6 & 60.8 & $\underline{1}$ \\
JOINT $_{\mathrm{kb}}$ & 60.0 & $\underline{58.8}$ & 61.1 & 0 \\
JOINTC $_{\mathrm{kb}}$ & $\underline{60.3}$ & $\underline{58.8}$ & $\underline{61.8}$ & 0 \\
\hline MAP $_{\text {hyb }}$ & 62.8 & 64.6 & 61.1 & 0 \\
JOINT $_{\text {hyb }}$ & 63.8 & 64.8 & 62.9 & 0 \\
JOINTC $_{\text {hyb }}$ & $\underline{\mathbf{6 5 . 3}}$ & $\underline{\mathbf{6 6 . 4}}$ & $\underline{\mathbf{6 4 . 3}}$ & $\underline{\mathbf{1 0}}$ \\
\hline
\end{tabular}

Table 9: Summary of results: average across all datasets and languages (all), average on relatedness datasets (rel), average on similarity datasets (sim) and number of best results across all datasets and languages (win). Underline for best in each group, and bold for best overall.

Table 8 presents the results of the three methods on the combined, hybrid, corpora. In this case, it is the joint method with bilingual constraints that consistently outperforms the other two methods in all datasets, showing that the constraints are effective in this setting and that our joint method is more effective than the mapping method. In fact, JOINTC $\mathrm{Chb}_{\mathrm{b}}$ yields the best results among all methods and embeddings (cf. Tables [5] and [6), showing that our method to combine text and knowledge bases is more effective to the state-ofthe-art mapping method.

\section{Summary of the main results and comparison to the state of the art}

Table 9 summarizes the most relevant results with four figures: average performance across all datasets and languages (all, 12 datasets), average performance on relatedness datasets (rel, average of 6 datasets), average performance on similarity datasets (sim, 6 datasets), and how many datasets does each 


\begin{tabular}{|l|l|ccc|}
\cline { 3 - 5 } \multicolumn{2}{c|}{} & WS & SL & RG \\
\hline \multirow{2}{*}{ ENES } & NASARI $_{\text {uni }}$ & 53.8 & 44.0 & 82.0 \\
& JOINTC $_{\text {hyb }}$ & $\mathbf{7 2 . 0}$ & $\mathbf{5 0 . 2}$ & $\mathbf{8 5 . 7}$ \\
\hline \multirow{2}{*}{ ENIT } & NASARI $_{\text {uni }}$ & 53.1 & $\mathbf{5 0 . 4}$ & - \\
& JOINTC $_{\text {hyb }}$ & $\mathbf{6 4 . 7}$ & 47.6 & - \\
\hline \multirow{2}{*}{ ESIT } & NASARI $_{\text {uni }}$ & 50.7 & $\mathbf{4 8 . 8}$ & - \\
& JOINTC $_{\text {hyb }}$ & $\mathbf{5 8 . 8}$ & 43.4 & - \\
\hline
\end{tabular}

Table 10: Results (Spearman) on cross-lingual datasets for the state-of-the-art NASARI unified (NASARI ${ }_{\text {uni }}$ ) and our system. The average performance are, respectively, 54.7 and 60.3, with our method winning in five out of the seven datasets.

method outperform the rest (win, 12 datasets). The summary shows clearly that our proposal to produce hybrid knowledge-based embeddings gets the best overall results, also in relatedness and similarity, and wins in 10 datasets out of the 12 .

In addition, Table 10 reports the results of the state-of-the-art NASARI which has been shown to outperform alternative methods in cross-lingual similarity [35]. We used the publicly available cross-lingual NASARI vectors that provided the best cross-lingual result: $15\left(\mathrm{NASARI}_{\mathrm{uni}}\right)$. They were readily available for English, Spanish and Italian, but the vectors for Basque were not available. We were generously assisted by one of the authors, so we could replicate their best results for English-Spanish and ensure that the rest of the results were correct. The table show that JOINTChyb beats NASARI in all English-Spanish similarity and relatedness datasets, also in English-Italian and Spanish-Italian relatedness, but not on the similarity datasets. The average performances are, respectively, 60.3 and 54.7, with our method winning in five out of the seven datasets. This is despite our algorithm using just wordnets, in contrast to the much richer BabelNet. In fact, our lower results for Italian could be partially

\footnotetext{
${ }^{15}$ http://lcl.uniroma1.it/nasari/\#two
} 
explained by the fact that the Italian wordnet is loosely aligned to the English WordNet, while BabelNet provides a tighter and richer integration. We think that our method combined with the richer BabelNet has the potential for further improvements.

We will now summarize the main findings in this article.

Random walks over multilingual wordnets improve results over just using dictionaries: According to our experiments, the state of the art in bilingual embeddings using monolingual corpora and bilingual dictionaries (row $\mathrm{MAP}_{\text {txt }}$ ) underperforms our best system ( JOINTC $_{\text {hyb }}$ ) in all datasets except one (WS for EUIT). Overall, we are able to improve results an average of 7 points, with the biggest gain on similarity (10 points, cf. Table 9). This shows that multilingual wordnets are not mere bilingual dictionaries, and that our random walk method is able to exploit the internal structure of wordnets.

Multilingual wordnets are specially strong on similarity: The information in the multilingual wordnets (without using textual corpora) is more effective than textual embeddings and bilingual dictionaries. Any of the methods to exploit random walks $\left(\mathrm{MAP}_{\mathrm{kb}}\right.$, JOINT $\mathrm{kb}$ or $\mathrm{JOINTC}_{\mathrm{kb}}$ ) beats MAP $\mathrm{Jxt}_{\mathrm{tn}}$ all similarity datasets, even if they do not exploit the information in textual corpora. The situation is reversed for word relatedness, where $\mathrm{KB}$ alone is slightly below, and in fact, the combination of wordnets and text is best for word relatedness.

The improvements work across all wordnets: The good results are notable for pairs with large wordnets like English and Spanish, but carry on to pairs with smaller wordnets like Basque, or loosely aligned wordnets like Italian. Note that the performance in ENIT is lower than in ENEU and ENES, either with our method or with MAP. This could be caused by the different structure of the Italian wordnet, and some information loss was to be expected because of the looser mapping. In the future, we would like to explore whether a different method to represent the graph for loosely aligned wordnets could be more effective.

Combining wordnets and textual corpora is very effective: From 
another perspective, it is thanks to the combination of textual corpora and knowledge bases, properly exploited by our random walk and joint learning method with added bilingual constraints (JOINTC $\mathrm{Chb}_{\mathrm{hb}}$ ) that we overperform the state-of-the-art in text-based bilingual embeddings ( $\mathrm{MAP}_{\mathrm{txt}}$ in Table 9). This fact is stressed by the fact that the joint method needs the synthetic corpora based on random walks to outperform MAP $_{t x t}$. Regarding the state of the art in hybrid knowledge- and text-based methods, we show that our embeddings overperform NASARI in 5 out of 7 datasets, even though our embeddings rely only on wordnets, in contrast to the richer BabelNet. The other two datasets involve the Italian wordnet, where our results are weaker (see above).

The two-step mapping method and our joint methods produce good results: All three methods, when applied to the hybrid corpora containing natural and synthetic text (MAPhyb, JOINThyb and JOINTChyb), are able to overperform the current state of the art, with our joint method using bilingual constraints providing the best results. When using monolingual text alone (three top lines in Table 9), the mapping method uses the bilingual dictionary more effectively than our joint method with constraints. When using the synthetic corpora, the situation is reversed, with the joint method providing better results, specially when combined with the bilingual constraints in the loss function. This might be caused by the strong signal in the bilingual synthetic corpora used by the joint method, which the joint method does not take profit from.

\section{Conclusions and future work}

Bilingual embeddings are typically produced using monolingual corpora to learn the embeddings and a bilingual dictionary to learn a mapping from one embedding space to the other. In this article we have proposed a novel method to produce a bilingual embedding space, based on monolingual corpora and bilingual wordnets. Bilingual wordnets can be used to produce bilingual dictionaries, but we propose a more effective method based on random walks that, in addition, extracts structural information. In fact we outperform the state of the 
art in bilingual embeddings [21], showing that we are able to make effective use of the structure of bilingual wordnets. Our approach is based on a random walk algorithm over bilingual wordnets which emits lexicalizations in two languages as it traverses the wordnets. The bilingual corpus produced in this manner is combined with monolingual corpora which is then fed into skipgram, yielding bilingual embeddings. Further improvements are obtained incorporating bilingual constraints extracted from the wordnets into the skipgram loss function.

From another perspective, we show that bilingual wordnets are more effective than bilingual dictionaries when producing bilingual embeddings. Given the increasing number of wordnets aligned with the English wordnet, and the fact that they can be used for any pair of languages which have a wordnet, our method shows great promise. For instance, larger bilingual knowledge bases like DBpedia or BabelNet could further augment the coverage of languages and further improve results. In fact, our method provides stronger results than NASARI 35] using just wordnets instead of the richer BabelNet, and it could be thus possible to improve further the results using richer resources. Domain-specific knowledge bases could be also useful when working on specific application areas. Note that, contrary to bilingual dictionaries and parallel corpora, once a wordnet is connected to the bilingual wordnets, it allows to produce bilingual embeddings of all other languages in the bilingual wordnet grid.

Our research opens a new avenue for producing multilingual embedding spaces, as it can work with larger Knowledge Bases like DBpedia or BabelNet, and can be easily extended from bilingual to multilingual embeddings. In addition to applying the method to other knowledge bases, we plan to explore further the different parameters of corpus combination. Being a first article using random walks for producing bilingual embeddings, we think there is still room for improvement. For instance, we would like to exploit multilingual wordnets and check whether multilingual embeddings for more than two languages are as effective as bilingual embeddings.

The software and embedding sets that performed best, as well as the cross lingual evaluation datasets and evaluation scripts are publicly available, with 
instructions to obtain out-of-the-box replicability 16. All software and resources of our method are open source.

\section{Acknowledgements}

We thank Mikel Artetxe and Jose Camacho-Collados for helping to run their systems, and Iraide Zipitria, Larraitz Uria and Izaskun Aldezabal for their contributions when building the Basque similarity datasets. This research was partially supported by a Google Faculty Research Award, the Spanish MINECO (TUNER TIN2015-65308-C5-1-R and MUSTER PCIN-2015-226) and the UP$\mathrm{V} / \mathrm{EHU}$ (excellence research group). Josu Goikoetxea enjoys a grant from the University of the Basque Country.

\footnotetext{
${ }^{16}$ url to be included upon acceptance
} 


\section{References}

[1] T. Mikolov, K. Chen, G. Corrado, J. Dean, Efficient estimation of word representations in vector space, arXiv preprint arXiv:1301.3781.

[2] T. Mikolov, I. Sutskever, K. Chen, G. S. Corrado, J. Dean, Distributed representations of words and phrases and their compositionality, in: Proceedings of Advances in neural information processing systems, 2013, pp. $3111-3119$.

[3] J. Pennington, R. Socher, C. D. Manning, Glove: Global vectors for word representation., in: Proceedings of EMNLP, Vol. 14, 2014, pp. 1532-1543.

[4] M. Baroni, G. Dinu, G. Kruszewski, Don't count, predict! a systematic comparison of context-counting vs. context-predicting semantic vectors., in: Proceedings of ACL (1), 2014, pp. 238-247.

[5] O. Levy, Y. Goldberg, Neural word embedding as implicit matrix factorization, in: Proceedings of Advances in neural information processing systems, 2014, pp. 2177-2185.

[6] D. Tang, F. Wei, N. Yang, M. Zhou, T. Liu, B. Qin, Learning sentimentspecific word embedding for twitter sentiment classification., in: Proceedings of ACL (1), 2014, pp. 1555-1565.

[7] K. M. Hermann, P. Blunsom, Multilingual models for compositional distributed semantics, in: Proceedings of the 52nd Annual Meeting of the Association for Computational Linguistics (Volume 1: Long Papers), Proceedings of ACL, Baltimore, Maryland, 2014, pp. 58-68.

URL http://www . aclweb.org/anthology/P14-1006

[8] S. R. Bowman, G. Angeli, C. Potts, C. D. Manning, A large annotated corpus for learning natural language inference, arXiv preprint arXiv:1508.05326. 
[9] G. Halawi, G. Dror, E. Gabrilovich, Y. Koren, Large-scale learning of word relatedness with constraints, in: Proceedings of the ACM SIGKDD, 2012, pp. $1406-1414$.

[10] M. Faruqui, J. Dodge, S. K. Jauhar, C. Dyer, E. Hovy, N. A. Smith, Retrofitting word vectors to semantic lexicons, in: Proceedings of the 2015 Conference of the North American Chapter of the Association for Computational Linguistics: Human Language Technologies, Association for Computational Linguistics, Denver, Colorado, 2015, pp. 1606-1615.

URL http://www.aclweb.org/anthology/N15-1184

[11] S. Rothe, H. Schütze, Autoextend: Extending word embeddings to embeddings for synsets and lexemes, in: Proceedings of the 53rd Annual Meeting of the Association for Computational Linguistics and the 7th International Joint Conference on Natural Language Processing (Volume 1: Long Papers), Proceedings of ACL, Beijing, China, 2015, pp. 1793-1803.

URL http://www . aclweb.org/anthology/P15-1173

[12] D. Bollegala, M. Alsuhaibani, T. Maehara, K.-i. Kawarabayashi, Joint word representation learning using a corpus and a semantic lexicon, in: Proceedings of AAAI, 2016, pp. 2690-2696.

[13] J. Goikoetxea, E. Agirre, A. Soroa, Single or multiple? combining word representations independently learned from text and wordnet., in: Proceedings of AAAI, 2016, pp. 2608-2614.

[14] G. A. Miller, Wordnet: a lexical database for english, Communications of the ACM 38 (11) (1995) 39-41.

[15] S. Gouws, Y. Bengio, G. Corrado, Bilbowa: Fast bilingual distributed representations without word alignments, in: Proceedings of the 32nd International Conference on Machine Learning, 2015, pp. 748-756.

[16] T. Luong, H. Pham, C. D. Manning, Bilingual word representations with monolingual quality in mind, in: 
Proceedings of the 1st Workshop on Vector Space Modeling for Natural Language Processing, Proceedings of ACL, Denver, Colorado, 2015, pp. $151-159$.

URL http://www.aclweb.org/anthology/W15-1521

[17] A. Søgaard, v. Agić, H. Martínez Alonso, B. Plank, B. Bohnet, A. Johannsen, Inverted indexing for cross-lingual nlp in: Proceedings of the 53rd Annual Meeting of the Association for Computational Linguistics and the 7th International Joint Conference on Natural Language Processing (Volume 1: Long Papers), Proceedings of ACL, Beijing, China, 2015, pp. $1713-1722$.

URL http://www . aclweb.org/anthology/P15-1165

[18] I. Vulic, M.-F. Moens, Bilingual distributed word representations from document-aligned comparable data Journal of Artificial Intelligence Research 55 (1) (2016) 953-994.

URL http://dl . acm.org/citation.cfm?id=3013558.3013583

[19] A. Mogadala, A. Rettinger, Bilingual word embeddings from parallel and non-parallel corpora for cross-la in: Proceedings of the 2016 Conference of the North American Chapter of the Association for Computational Linguistics: Human Language Technologies, Proceedings of ACL, San Diego, California, 2016, pp. 692-702.

URL http://www.aclweb.org/anthology/N16-1083

[20] T. Mikolov, W.-t. Yih, G. Zweig, Linguistic regularities in continuous space word representations, in: Proceedings of HLT-NAACL, 2013, pp. 746-751.

[21] M. Artetxe, G. Labaka, E. Agirre, Learning principled bilingual mappings of word embeddings while prese in: Proceedings of EMNLP, Association for Computational Linguistics, Austin, Texas, 2016, pp. 2289-2294.

URL https://aclweb.org/anthology/D16-1250

[22] C. Xing, D. Wang, C. Liu, Y. Lin, Normalized word embedding and orthogonal transform for bilingual word translation., in: Proceedings of HLTNAACL, 2015, pp. 1006-1011. 
[23] P. Vossen, A multilingual database with lexical semantic networks, Springer, 1998.

[24] J. Goikoetxea, A. Soroa, E. Agirre, B. C. Donostia, Random walks and neural network language models on knowledge bases., in: Proceedings of HLT-NAACL, 2015, pp. 1434-1439.

[25] R. Navigli, S. P. Ponzetto, Babelnet: The automatic construction, evaluation and application of a wide-co Artif. Intell. 193 (2012) 217-250. doi:10.1016/j.artint.2012.07.001. URL http://dx.doi.org/10.1016/j.artint.2012.07.001

[26] J. Ganitkevitch, B. Van Durme, C. Callison-Burch, Ppdb: The paraphrase database., in: Proceedings of HLT-NAACL, 2013, pp. 758-764.

[27] K. Bollacker, C. Evans, P. Paritosh, T. Sturge, J. Taylor, Freebase: a collaboratively created graph database for structuring human knowledge, in: Proceedings of the ACM SIGMOD, 2008, pp. 1247-1250.

[28] C. F. Baker, C. J. Fillmore, J. B. Lowe, The berkeley framenet project, in: Proceedings of the 36th Annual Meeting of the Association for Computational Linguistics and 17th International Conference on Computational Linguistics-Volume 1, Proceedings of ACL, 1998, pp. 86-90.

[29] P. Rastogi, B. Van Durme, R. Arora, Multiview LSA: Representation Learning via Generalized CCA, in: Proceedings of HLT-NAACL, 2015, pp. $556-566$.

[30] Z. Wang, J. Zhang, J. Feng, Z. Chen, Knowledge graph embedding by translating on hyperplanes., in: Proceedings of AAAI, Citeseer, 2014, pp. $1112-1119$.

[31] B. Perozzi, R. Al-Rfou, S. Skiena, Deepwalk: Online learning of social representations, in: Proceedings of the 20th ACM SIGKDD International Conference on Knowledge Discovery and Data Mining, KDD '14, ACM, New York, NY, USA, 2014, pp. 701-710. doi:10.1145/2623330.2623732.

URL http://doi.acm.org/10.1145/2623330.2623732 
[32] T. Mikolov, Q. V. Le, I. Sutskever, Exploiting similarities among languages for machine translation, arXiv preprint arXiv:1309.4168.

[33] Y. Zhang, D. Gaddy, R. Barzilay, T. Jaakkola, Ten pairs to tag-multilingual pos tagging via coarse mapping between embeddings, in: Proceedings of the 2016 Conference of the North American Chapter of the Association for Computational Linguistics: Human Language Technologies, 2016, pp. $1307-1317$.

[34] M. Faruqui, C. Dyer, Improving vector space word representations using multilingual correlation, in: Proceedings of the 14th Conference of the European Chapter of the Association for Computational Linguistics, Association for Computational Linguistics, Gothenburg, Sweden, 2014, pp. 462-471.

URL http://www . aclweb.org/anthology/E14-1049

[35] J. Camacho-Collados, M. T. Pilehvar, R. Navigli, Nasari: Integrating explicit knowledge and corpus statistics for a multilingual representation of concepts and entities, Artificial Intelligence 240 (2016) 36-64.

[36] O. Levy, Y. Goldberg, I. Dagan, Improving distributional similarity with lessons learned from word embeddings, Proceedings of TACL 3 (2015) 211225.

[37] M. Wick, P. Kanani, A. Pocock, Minimally-constrained multilingual embeddings via artificial code-switching., in: Proceedings of AAAI, 2016, pp. 2849-2855.

[38] E. Agirre, E. Alfonseca, K. Hall, J. Kravalova, M. Paşca, A. Soroa, A study on similarity and relatedness using distributional and WordNet-based approaches, in: Proceedings of of HLT-NAACL, 2009, pp. 19-27.

[39] T. Luong, R. Socher, C. D. Manning, Better word representations with recursive neural networks for morphology., in: Proceedings of CoNLL, 2013, pp. $104-113$. 
[40] I. Leturia, Evaluating different methods for automatically collecting large general corpora for basque from the web., in: Proceedings of COLING, 2012, pp. 1553-1570.

[41] A. G. Agirre, E. Laparra, G. Rigau, B. C. Donostia, Multilingual central repository version 3.0: upgrading a very large lexical knowledge base, in: GWC 2012 6th International Global Wordnet Conference, 2012, p. 118.

[42] A. Roventini, A. Alonge, F. Bertagna, N. Calzolari, J. Cancila, C. Girardi, B. Magnini, R. Marinelli, M. Speranza, A. Zampolli, Italwordnet: building a large semantic database for the automatic treatment of italian, Linguistica Computazionale, Special Issue (XVIII-XIX) (2003) 745-791.

[43] E. Gabrilovich, S. Markovitch, Computing Semantic Relatedness Using Wikipedia-based Explicit Semantic Analysis., in: Proceedings of IJCAI, Vol. 7, 2007, pp. 1606-1611.

[44] F. Hill, R. Reichart, A. Korhonen, Simlex-999: Evaluating semantic models with (genuine) similarity estimation, Computational Linguistics.

[45] H. Rubenstein, J. B. Goodenough, Contextual correlates of synonymy, Communications of the ACM 8 (10) (1965) 627-633.

[46] J. Camacho-Collados, M. T. Pilehvar, R. Navigli, A framework for the construction of monolingual and cross-lingual word similarity datasets., in: Proceedings of ACL (2), 2015, pp. 1-7.

[47] S. Hassan, R. Mihalcea, Cross-lingual semantic relatedness using encyclopedic knowledge, in: Proceedings of the 2009 Conference on Empirical Methods in Natural Language Processing: Volume 3-Volume 3, Proceedings of ACL, 2009, pp. 1192-1201.

[48] M. Etcheverry, D. Wonsever, Spanish word vectors from wikipedia, in: N. C. C. Chair), K. Choukri, T. Declerck, S. Goggi, M. Grobelnik, B. Maegaard, J. Mariani, H. Mazo, A. Moreno, J. Odijk, S. Piperidis (Eds.), 
Proceedings of the Tenth International Conference on Language Resources and Evaluation (LREC 2016), European Language Resources Association (ELRA), Paris, France, 2016, pp. 3681-3685.

[49] M. Baroni, G. Dinu, G. Kruszewski, Don't count, predict! a systematic comparison of context-counting vs. context-predicting semantic vector in: Proceedings of the 52nd Annual Meeting of the Association for Computational Linguistics (Volume 1: Long Papers), Proceedings of ACL, Baltimore, Maryland, 2014, pp. 238-247.

URL http://www . aclweb.org/anthology/P14-1023 